Kenntnis gebracht werden würde. In dieser Beziehung kommt folgender beachtlicher Punkt noch in Frage: Frische Eier oder frisches Eigelb können wegen ihres hohen Marktpreises angeblich nicht mehr zur Herstellung von Mayonnaisen verwendet werden. An ihrer Stelle wird meist Trockeneigelb benutzt, das erfahrungsgemäß fast alle Quellungs- und Bindekraft eingebüßt hat. An Stelle des Eigelbes, auch wenn seine Menge an und für sich genügen würde (entsprechend etwa 3-4 Eiern auf 11 Öl), muß ein anderes Mittel zugesetzt werden, um die Bindung zwischen Eigelb und Öl hervorzurufen, nämlich Mehl. Man wird sagen dürfen, daß solche mit Trockeneigelb und unter Mehlzusatz hergestellten Mayonnaisen von der normalen, bisher gewerbegerecht hergestellten Ware abweichen und daher auch dann, wenn sie keine übermäßige Menge Wasser entbalten, als nachgemacht im Sinne des Nahrungsmittelgesetzes anzusehen sind. Bei entsprechender Kennzeichnung des Zusatzes von Trockeneigelb und $\mathrm{Mehl}$, welch letzteres eine bestimmte Höhe nicht überschreiten sollte (etwa $2 \%$, wäre unseres Erachtens gegen den Verkauf solcher Ware nichts einzuwenden.

\title{
Die Einwirkung des Babassufutters auf Butter- und Schweinefett.
}

\author{
Von \\ N. Bengtsson.
}

Mitteilung aus dem Bakteriologischen Laboratorium der Zentralanstalt für landwirtschaftliches Versuchswesen auf Experimentalfaltet bei Stockholm.

\section{[Eingegangen am 14. November 1922.]}

Bei der Fütterung von fettreichen pflanzlichen Futtermitteln ist die Frage von besonderem Interesse, ob diese etwa einen Einfluß auf die physikalischen und analytischen Konstanten der damit erzeugten Fettarten ausüben. Infolgedessen baben wir einige Untersuchungen bei Butter- und Schweinefetten ausgeführt, die nach der Fütterung mit Babassukuchen gewonnen worden waren.

Die Babassukuchen waren aus Samen der südamerikanischen Palmenart Orbignya Lydia Dr. (syn. Attalea speciosa Mart.) hergestellt worden.

Die klaren, filtrierten Fettproben wurden auf ihre Reichert-Meißl'sche Zahl, Polenske'sche Zahl, Jodzahl, Verseifungszahl, Refraktometerzahl und Schmelzpunkt untersucht. Die Bedeutung dieser Konstanten und die Methoden zu ihrer Bestimmung sind in Chr. Barthel: „Die Methoden zur Untersuchung von Milch und Molkereiprodukten“ (3. Aufl., Verlag von Pa ul Parey, Berlin 1920), beschrieben. Zu den Fällen, wo in diesem Buche mehrere Bestimmungsmethoden angegeben sind, sei mitgeteilt, daß die Refraktometerzahl (bei $40^{\circ}$ ) mit dem Wollny-Zei $@$ Butterfettrefraktometer bestimmt wurde, und daß die Jodzahl nach dem Bromaddierungsverfahren von $W$ inkler ermittelt wurde.

\section{Butteruntersuchungen.}

Die Butterproben stammten aus einem Fütterungsversuche mit Milchkühen (Gruppen I und II), die in den Jahren 1920-1921 ausgeführt wurden. Die Perioden und Mengen der Babassugahen dieser Gruppen waren folgende: 
Es erhielten je Tier und Tag:

Gruppe I. 23. XII.-30. XII. 20: 0,10 kg Babassukuchen,

31. XII. 20-2. I. 21: $0,50 \mathrm{~kg} \quad "$

3. I. $21-14$. I. $21: 0,96 \mathrm{~kg} \quad "$

worauf die Babassakuchen durch Erbsen ersetzt wurden.

Gruppe II. 23. XII. 20-14. I. 21: Dieselbe Fütterung wie Gruppe I, 15. I. $21-18$. II. $21: \quad 1,20 \mathrm{~kg}$ Babassakuchen,

worauf die Fütterung mit Babassakuchen wegfiel.

Die Ergebnisse der Butterfettuntersuchungen sind in Tabelle I zusammengestellt. Die darin mitgeteilten Ergebnisse sind, abgesehen von den Refraktometerzahlen, für welche Einzelbestimmungen genügend angesehen wurden, ebenso wie bei den später behandelten Schweinefettuntersuchungen, das Mittel von zwei gut übereinstimmenden Werten.

Tabelle I. Butterfettuntersuchangen.

\begin{tabular}{c|c|c|c|c|c|c|c|c}
\hline Nr & $\begin{array}{c}\text { Versuchs. } \\
\text { gruppe }\end{array}$ & $\begin{array}{c}\text { Tag } \\
\text { der Ent- } \\
\text { rahmung }\end{array}$ & $\begin{array}{c}\text { Reichert } \\
\text { Meikl'. } \\
\text { sche Zaht }\end{array}$ & $\begin{array}{c}\text { Polens- } \\
\text { ke'sche } \\
\text { Zahl }\end{array}$ & Jodzahl & $\begin{array}{c}\text { Ver- } \\
\text { seifungs- } \\
\text { zahl }\end{array}$ & $\begin{array}{c}\text { Refrakto- } \\
\text { meterzahl }\end{array}$ & $\begin{array}{c}\text { Schmelz- } \\
\text { punkt }\end{array}$ \\
\hline $\mathbf{1}$ & I & 14. I. 21 & 28,8 & 3,0 & 30,6 & 235,2 & 42,3 & 32,6 \\
2 & II & 14. I. 21 & 28,8 & 3,0 & 32,1 & 234,2 & 42,3 & 32,7 \\
3 & I & 5. II. 21 & 28,8 & 3,1 & 31,3 & 231,8 & 42,0 & 33,7 \\
4 & II & 5. II. 21 & 27,3 & 3,3 & 33,2 & 230,0 & 41,8 & 32,5 \\
5 & I & 14. III. 21 & 28,9 & 2,75 & 29,0 & 229,5 & 41,9 & $\mathbf{3 3 , 3}$
\end{tabular}

Die Proben Nr. 1 und 2 der Gruppen I und II, welche bis zum Tage der Probenahme dieselbe Fütterung, worin auch Babassakuchen enthalten waren, erhielten, beweisen die große Übereinstimmung zwischen den beiden Versuchsgruppen, da ja eigentlich nur die Jodzahlen eine unwesentliche Verschiedenheit aufweisen. Probe Nr. 5 kann offenbar als Kontrolle angesehen werden, da sie so spät nach der Beendigung der Babassufütterung genommen ist, daß jede Nachwirkung dieser Fütterung als ausgeschlossen betrachtet werden kann. Der Einfluß der Babassukuchen auf die Konstanten der übrigen Proben geht mithin aus der Differenz zwischen diesen und den Konstanten der Probe Nr. 5 hervor.

Über die Konstanten des Babassufettes, welches der einzige Bestandteil der Babassukuchen ist, wovon eine Einwirkung auf die Konstanten der produzierten Fette erwartet werden kann, macht W. Clayton in seinem Werke "Margarine“ (Verlag von Longmans, Green and Co., London 1920, S. 7) folgende Angaben:

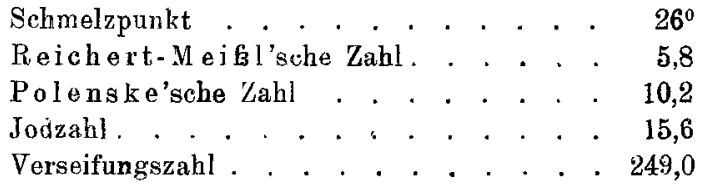

Nach den Untersuchungen des Laborators H. Edin an der Haustierzuchtabteilung der Zentralanstalt, welcher die hier erwähnten Fütterungsversuche ausgeführt hat, enthielten die Babassukuchen 17,27\% Rohfett (Mittel von drei Bestimmungen); sie hatten also einen ungewöhnlich hohen Fettgebalt. 
Die Proben Nr. 1, 2 und 3 zeigen dieselbe Reichert-Meißl'sche Zabl wie die Kontrollprobe, dagegen haben sie eine wesentlich höhere Polenske'sche Zahl als diese. Bei Probe Nr. 4 ist die Zunahme der Polenske'schen Zahl noch größer, während die Reichert-M eißl'sche Zahl wiederum erniedrigt ist. Polenske hat bekanntlich gefunden, daß bei natürlicher Butter eine Beziehung zwischen der Reichert. Meißl'schen Zahl und der Polenske'schen Zahl besteht, und daß ein Überschreiten der „höchstzulässigen“ Polenske'schen Zahl ein Kennzeichen für die Beimischung von Cocosfett ist.

Für Butterfette mit den Reichert-Meißl'schen Zahlen 27-29 sind die Beziehungen folgende:

Reichert-Meibl'sche
Zahl
$27-28$
$28-29$

\section{Polenske'sche Zahl}

$2,0-2.2$ $2,2-2,5$

\author{
Höchstzulässige \\ Polenske'sche Zahl
}

2,7

3,0

Die Polenske'schen Zahlen der Proben Nr. 1, 2 und 3 fallen also mit den böchstzulässigen Polenske'schen Zahlen zusammen, und bei der Probe Nr. 4 wird dieselbe beträchtlich überschritten. Eine mittelgroße Babassugabe erhöht mithin die Polenske'sche Zahl bis an die böchstzulässige Grenze (Probe Nr. 1 u. 2), welche Einwirkung noch drei Wochen nach dem Aufhören der Babassufütterung an bält (Probe Nr. 3), und nach einer starken Babassugabe wird der Grenzwert überschritten (Probe Nr. 4). Lührig, Siegfeld, Ledent wie auch Barthel und Sondén ${ }^{5}$ ) haben früher solche zu hohen Polenskeschen Zahlen bei Butter nach der Fütterung mit Cocoskuchen beobachtet. Die Jodzahlen sind bei den sämtlichen Babassuproben erhöht, aber nur bei der höchsten Gabe (Probe Nr. 4) ist die Erhöhung von Betracht. Auch die Verseifungs- und Refraktometerzahlen zeigen gegenüber der Kontrollprobe eine Erhöhung, aber diese ist nicht auf die Babassukuchen zurückzuführen, sondern muß zufälliger Natur sein. Der Schmelzpunkt dagegen ist von dem Babassufutter deutlich beeinflusst, indem er bei den Proben Nr. 1 und 2 und besonders bei der Probe Nr. 4 einen entschieden niedrigeren Wert aufweist als bei der Kontrollprobe. Diese Einwirkung hört indessen schon innerhalb drei Wochen nach der Beendung der Babassufütterung auf (Probe Nr. 3). Die Richtigkeit der Schmelzpunktbestimmungen bei den Proben Nr. 3 und 4 wurde bestätigt durch eine Beurteilung der entsprechenden Butterproben, welche Oberassistent E. Haglund an der Milchwirtschaftlichen Abteilung der Zentralanstalt am 26. Februar 1921 ausführte, wobei die Butterprobe Nr. 311 Punkte erbielt, während die Probe Nr. 4 10,5 Punkte mit der Bemerkung ,schmierig“ erhielt.

\section{Schw einefettuntersuchungen.}

Die Schweinefettuntersuchungen beziehen sich auf Schmalz und Speck von einem Fütterungsversuche mit Babassukuchen, der vom Februar-Mai 1921 ausgeführt wurde, wobei die eine Gruppe (I) mit Babassukuchen, die zweite Gruppe (II) ob ne Babassukuchen gefüttert wurde. Die Babassugaben der Gruppe I waren folgende je Tier und Tag: 
5. II. bis 11. Il. $21: 0,29 \mathrm{~kg}$

12. II. , 18. I1. $21: 0,46$,

19. II., 25 . II. $21: 0,50$,

26. II. \# 4. IIL. $21: 0,62$,

5. III. " 11. III. $21: 0,61$,
12. III. bis 18. IIII. $21: 0,77 \mathrm{~kg}$

19. III. * 25. III. $21: 0,86$,

26. III. " 15. IV. $21: 0,93$,

16. IV. " 20. V. $21: 1,00$,

worauf die Schlachtung erfolgte.

Bei diesen Untersuchungen wurde von Bestimmungen der Reichert-MeiBlschen und Polenske'schen Zahlen abgesehen, weil man kaum irgend eine Einwirkung auf diese Konstanten erwarten kann, da sie bei Tierkörperfetten nur einen sehr niedrigen Wert haben, der bei wechselnder Fütterung nur einen unbedeutenden Ausschlag zeigt. Im übrigen wurden dieselben Bestimmungen wie bei den Butterfetten ausgeführt. Die Ergebnisse der Schweinefettanalysen sind in Tabelle II wiedergegeben:

Tabelle II. Schweinefettuntersuchungen.

\begin{tabular}{|c|c|c|c|c|c|c|c|}
\hline $\begin{array}{c}\mathrm{Nr} . \\
\text { der } \\
\text { Frobe }\end{array}$ & Fütterung & & Fettart & Jodzahl & $\begin{array}{c}\text { Ver- } \\
\text { seifungs- } \\
\text { zahl }\end{array}$ & $\begin{array}{l}\text { Refrakto- } \\
\text { meterzabl }\end{array}$ & $\begin{array}{l}\text { Sehmelz- } \\
\text { punkt }\end{array}$ \\
\hline 6 & Mit Babassukuchen & . & Schmalz & 51,2 & 195,0 & 48,4 & 47,2 \\
\hline 7 & Ohne & . & $\pi$ & 53,7 & 194,8 & 48,9 & 44,5 \\
\hline 8 & Mit & . . & Speck & 56,5 & 195,8 & 49,1 & 44,0 \\
\hline 9 & Ohne & . : & $n$ & 60,2 & 192,7 & 49,9 & 43,6 \\
\hline
\end{tabular}

Aus dieser Tabelle geht hervor, daß die erhaltenen Ergebnisse bei den Schmalzund Speckfetten durchgehends nach derselben Richtung hindeuten. Im Gegensatz zu den Butterfetten hat die Babassufütterung hier eine Erniedrigung der Jodzahl bewirkt, welche ziemlich beträchtlich ist. Dagegen zeigen die Verseifungszahlen keine nennenswerte Beeinflussung durch die Babassukuchenfütterung. Den Refraktometerzahlen und Schmelzpunkten dagegen hat die Fütterung mit Babassukuchen eine entgegengesetzte Richtung gegeben, indem die Refraktometerzahlen sinken, die Schmelzpunkte dagegen steigen. Die eingetretenen Veränderungen sind jedoch in keinem Falle so groß, daß die betreffenden Konstanten außerhalb der normalen Grenzen zu liegen kommen.

Das Ergebnis der beschriebenen Untersuchungen ist, daß die Fütterung mit Babassukuchen beim Butterfett die Reichert-Meißl'sche Zahl und die Polenske'sche Zahl in der Richtung verschiebt, daß man die Butter, ohne eingehende Untersuchung einer Verfälschung mit Pflanzenfett verdächtig ansehen mub. Ohne nähere Kenntnis von einer derartigen Fütterung würde man in einem solchen Falle, um Gewißheit in diesem Punkte zu erhalten, die Phytosterinacetatprobe ausfübren müssen. Im vorliegenden Falle war eine Untersuchung nach dieser Richtung natürlich nicht erforderlich. 